Research paper

\title{
Supramolecular cyclodextrin-based metal-organic frameworks as efficient carrier for anti-inflammatory drugs
}

\author{
Marina P. Abuçafy ${ }^{a}$, Bruno L. Caetano ${ }^{a}$, Bruna G. Chiari-Andréo ${ }^{a, b}$, Bruno Fonseca-Santos ${ }^{a}$, \\ Aline M. do Santos ${ }^{\mathrm{a}}$, Marlus Chorilli ${ }^{\mathrm{a}}$, Leila A. Chiavacci ${ }^{\mathrm{a}, *}$ \\ ${ }^{a}$ Department of Drugs and Medicines, São Paulo State University (UNESP), School of Pharmaceutical Sciences, Araraquara, SP, Brazil \\ ${ }^{\mathrm{b}}$ Department of Biological and Health Sciences, Universidade de Araraquara - UNIARA, Araraquara, SP, Brazil
}

\section{A R T I C L E I N F O}

\section{Keywords:}

Metal-organic framework

$\gamma$-cyclodextrin

Drug adsorption

Drug delivery

Anti-inflammatory in vivo assay

\begin{abstract}
A B S T R A C T
Drug delivery systems have been used to reduce adverse effects and improve the efficacy of therapies. Drug carriers have been developed over the years, but they have limitations. $\gamma$-cyclodextrin-based metal-organic frameworks ( $\gamma$-CD-MOF) have significant advantages due to their biocompatibility and environmental safety, besides crystallinity and porosity. Herein, $\gamma$-CD-MOFs were synthesised with different metals as nodes and investigated. Uniform mesoporous $\gamma$-CD-MOFs were obtained and showed an absence of toxicity in HepG2 and Caco- 2 cells. The longer controlled release was verified for $\gamma$-CD-MOFs, with a maximum of $62 \%$ released in $12 \mathrm{~h}$. An inflammation experiment was performed in mice and activity equivalent to the positive control was verified. $\gamma$-KCD-MOFs and $\gamma$-NaCD-MOFs reached activity after $6 \mathrm{~h}$ of administration, however this happened after $24 \mathrm{~h}$ in $\gamma$-FeCD-MOFs, being more effective than the positive control. Considering the ability for drug entrapment, easy preparation and controlled release, this class of material allows potential applications in drug delivery systems.
\end{abstract}

\section{Introduction}

Metal-organic frameworks (MOFs) are a new class of hybrid materials composed of metal centres (nodes) and organic linkers (spacers). The framework formed is crystalline and highly porous, with a tuneable pore shape, size and functionality [1,2], which have aroused increasing attention due to several potential applications in the strategic field, such as catalysis [3], gas storage [4,5], separation science [6,7] and, more recently, biomedical approaches concerning drug delivery [8].

Considering MOFs as promising alternative drug delivery systems, they can be employed aiming to overcome the characteristics of polymeric and mesoporous silica systems, such as the storage capacity and the control of drug release. Their high and regular porosity combined with their crystallinity could contribute to high drug loading, as well as to a more controlled release in simulated physiological conditions [9].

Also, drug delivery systems need to be biocompatible; however, the vast majority of recently developed MOFs were non-renewable, which the metal nodes and organic linkers used were toxic, making these materials unsuitable for biomedical applications [10]. Thus, the development of MOFs using biologically acceptable metals, such as $\mathrm{Ca}, \mathrm{Fe}$, $\mathrm{Zn}, \mathrm{K}, \mathrm{Na}$ and $\mathrm{Ti}$, and natural linkers such as peptides [8], carbohydrates [11] and amino acids [12] has recently increased.

A new strategy to overcome this problem of toxicity and maintain crystallisation as a porous framework has been reported using gamma cyclodextrin $(\gamma-\mathrm{CD})$ as an organic linker [10,13-15]. Cyclodextrin is a symmetrical cyclic oligosaccharide, which is produced enzymatically from starch [16] and has asymmetric 1,4-linked alpha glucopyranosyl residues that form a body-centred cubic structure when linked with the metal cations by the coordination of -OCCO- units.

The $\gamma$-CD-MOF became popular due to their biocompatibility and potential application for entrapment molecules and for biomedical purposes. Despite this growing interest in the development of "green" MOFs with cyclodextrin, there has not been much effort with regard to drug delivery systems $[17,18]$. We describe here a method for the encapsulation and controlled release of an anti-inflammatory drug model, sodium diclofenac (DFNa), by different MOFs with $\gamma$-cyclodextrin $(\gamma$ CD-MOF): $\gamma$-KCD, $\gamma$-NaCD and $\gamma$-FeCD. The porosity and crystallinity of these materials based on biocompatible components allow their use as drug delivery systems to be illustrated.

The in vitro cytotoxicity assay, in vitro drug release, and in vivo antiinflammatory activity were investigated and showed the promising property of this material for pharmaceutical applications.

\footnotetext{
* Corresponding author.

E-mail address: leila@fcfar.unesp.br (L.A. Chiavacci).
} 


\section{Experimental section}

\subsection{Materials}

All chemicals were purchased from Sigma-Aldrich, with the exception of sodium diclofenac, which was purchased from Audaz. Dichloromethane and methanol were purchased from Lac. Ferric chloride $\mathrm{Fe}_{2} \mathrm{Cl}_{3}$ (97\%) was purchased from Dinâmica Química Contemporânea Ltda.

\subsection{Synthesis of $\gamma$-CD-MOFs}

Cyclodextrin-based metal-organic frameworks ( $\gamma$-CD-MOFs) were obtained by the modified vapour diffusion method, which has been previously reported [13,17-19]. The crystals were prepared by reacting $\gamma$-CD $(1 \mathrm{mmol})$ with a solution of metal $(8 \mathrm{mmol})$, derived from metal salts such as potassium hydroxide, sodium hydroxide and iron chloride, in $20 \mathrm{~mL}$ of aqueous solution. The solution was filtered into a glass tube followed by methanol diffusion at room temperature for 7 days. The preferential volatilisation of methanol, which is the liquid with the lowest boiling, enriches the chamber saturation with vapours which slowly diffuse into the polar solution, containing metal and organic linkers, gradually decreasing the polarity of the solution and reducing the solubility of the sample, leading to the slow formation of crystals [15]. After that, the solid product was filtered, washed with methanol three times and then activated by solvent-exchange. The activation is a process that is capable of unblocking pores via the removal of residual solvent; this process was first demonstrated as solvent-exchange by $\mathrm{Li}$ et al. [19]. The activation by solvent-exchange was carried out by submerging $\gamma$-CD-MOF crystals in dichloromethane $\left(\mathrm{CH}_{2} \mathrm{Cl}_{2}\right)$ for $24 \mathrm{~h}$. In this step, the residual methanol is exchanged for dichloromethane, a solvent with a lower boiling point, followed by vacuum drying in a heating oven at $50{ }^{\circ} \mathrm{C}$ for a further $24 \mathrm{~h}$. Fig. 1 shows a schematic representation of the vapour diffusion method for the synthesis of $\gamma$-CDMOFs.

The yield of $\gamma$-CD-MOF synthesis is defined as the ratio between the amount of $\gamma$-CD-MOF formed and the amount of cyclodextrin and metal salt added during the preparation procedure. The solid material was dried and the yield $(Y)$ was calculated using the following Eq. (1):

$Y(\%)=\left(\frac{\text { weight of } \gamma \text {-CD-MOF prepared }}{\text { total weigh of reagents }}\right) \times 100$

\section{3. $X$-ray diffraction $(X R D)$}

XRD patterns were analysed in a conventional high resolution X-ray diffractometer (Siemens D5000) using $\mathrm{Cu} \mathrm{Ka}(\lambda=1.54 \AA$ ) with a step size of $0.02^{\circ} \mathrm{o}$ and $4 \mathrm{~s}$ per step in a continuous mode.

\subsection{Infrared (IR)}

$\gamma$-CD and $\gamma$-CD-MOFs samples were examined using a Nicolet IS5 IR spectrophotometer (Thermo Scientific, Pittsburgh, PA, USA). The wavenumber range was 400 to $4000 \mathrm{~cm}^{-1}$ using $\mathrm{KBr}$ pellets.

\subsection{Thermogravimetric analysis (TGA)}

Samples were analysed under nitrogen ( $\left.50 \mathrm{~mL} \cdot \mathrm{min}^{-1}\right)$ using a simultaneous DTA/TGA of TA Instruments SDT 600 running from room temperature to $800^{\circ} \mathrm{C}$ with a heating rate of $5^{\circ} \mathrm{C} \cdot \mathrm{min}^{-1}$.

\subsection{Nitrogen adsorption-desorption}

$\mathrm{N}_{2}$ adsorption-desorption of the crystal samples was measured with a liquid $\mathrm{N}_{2}$ bath $\left(-196^{\circ} \mathrm{C}\right)$ using a Micromeritics ASAP (Accelerated Surface Area and Porosimetry System) porosimeter. Known amounts of sample were loaded into BET sample tubes and degassed under vacuum $\left(10^{-4}\right.$ Torr $)$ at $100{ }^{\circ} \mathrm{C}$ for $3 \mathrm{~h}$. The BET model was applied to measure the pore size.

\subsection{Scanning electron microscopy (SEM)}

$\gamma$-CD-MOF samples were coated with gold with an approximate thickness of $20 \mathrm{~nm}$ on the surface for $80 \mathrm{~s}$ and SEM was analysed by the TopconSM-300 with the detection of secondary electrons using an electron accelerating voltage of $10 \mathrm{kV}$.

\subsection{Cytotoxicity assay}

$\gamma$-CD-MOF cytotoxicity was assessed on HepG2 (human hepatoma) and Caco-2 (human epithelial colorectal adenocarcinoma) cells. The cell cultures were maintained in DMEM (Dulbecco's Modified Eagle's medium) $\mathrm{pH} 7.4$, supplemented with $10 \%$ foetal bovine serum and antibiotics (penicillin $100 \mathrm{U} / \mathrm{ml}$, streptomycin $0.1 \mathrm{mg} / \mathrm{ml}$ ). The culture bottles were maintained in an incubator at $37^{\circ} \mathrm{C}$ with $5 \% \mathrm{CO}_{2}$. Then, 3-9 passage-numbers were used to perform the experiments and the cells were plated on 96-well flat-bottom microplates at a density of about $10^{5}$ cells $/ \mathrm{mL}$. Seven MOF concentrations (ranging from 10 to $2000 \mu \mathrm{g} / \mathrm{mL}$ ) were made by serial dilutions of $\gamma$-CD-MOF in DMEM. This range of concentrations was determined as the maximum amount able to be solubilised in the culture media. The cells were treated with $100 \mu \mathrm{L}$ of each concentration of $\gamma$-CD-MOF suspension with DMEM. Also, a negative control (DMEM) and a positive control (10\% DMSO) were assayed. After $24 \mathrm{~h}$ of incubation, the samples were removed and cells were incubated with $100 \mu \mathrm{L}$ MTT [3-(4,5-dimethylthiazol-2-yl)2,5-diphenyltetrazoliumbromide] $(1 \mathrm{mg} / \mathrm{mL})$ solution in Phosphate Buffered Saline (PBS) for 3-5h. Subsequently, the MTT solution was a)

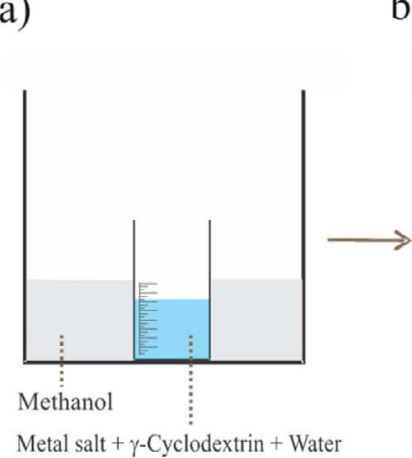

b)

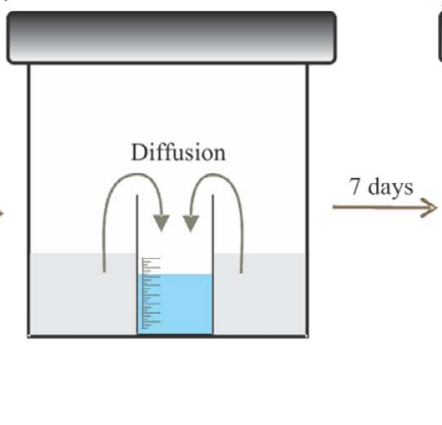

c)

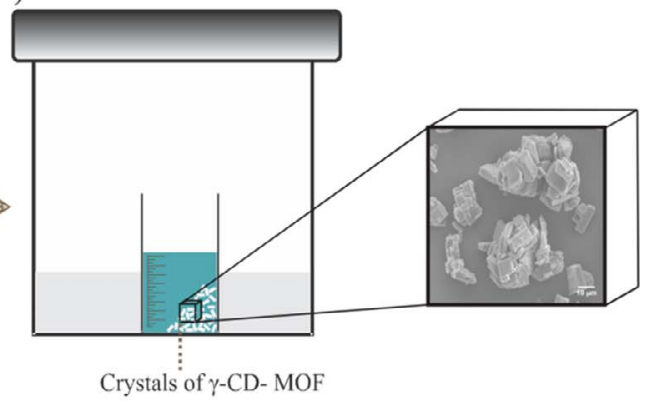

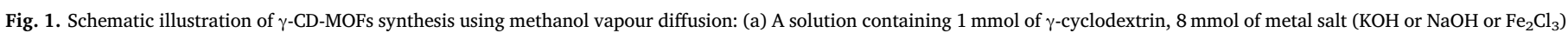

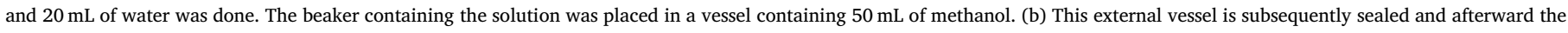
methanol, that it is more volatile, diffuses into the inner beaker containing the solution. (c) After a few days crystals were formed and an image of MEV is shown in the zoom. 
removed and $50 \mu \mathrm{L}$ of isopropyl alcohol was added to solubilise the formazan crystals. After $10 \mathrm{~min}$, the absorbance of each well was measured at $595 \mathrm{~nm}$ by a plate reader (iMarkMicroplate Absorbance Reader, model 168-1135, Bio-Rad, USA). All samples were tested in triplicate for each assay. The cytotoxicity was calculated by comparing changes between the negative control and treated cells and data are represented as mean \pm standard error.

\subsection{Entrapment efficiency}

The loading of drugs was carried out after the activation of $\gamma$-CDMOF by the impregnation approach and DFNa was used as a model drug. Impregnation was performed by suspending $60 \mathrm{mg}$ of $\mathrm{MOF}$ powder in $42 \mathrm{~mL}$ of ethanol DFNa solution under stirring at room temperature for $72 \mathrm{~h}$. The loaded drugs were recovered by centrifugation $(4000 \mathrm{rpm}, 10 \mathrm{~min})$ and dried at $35^{\circ} \mathrm{C}$ overnight under vacuum. The drug in the supernatant was determined by UV/Vis spectroscopy (Agilent Cary 60Spectrophotometer, Australia), at $275 \mathrm{~nm}$, in triplicate. The entrapment efficiency (EE\%) was calculated according to the following Eq. (2):

Entrapment efficiency $(\% \mathrm{EE})=\frac{\text { weight of drug in } \gamma \text {-CD-MOF }}{\text { weight of drug fed initially }} \times 100$

\subsection{In vitro release studies}

The in vitro release profile of DFNa from DFNa-loaded- $\gamma$-CD-MOF samples was determined by the dialysis method [20]. $10 \mathrm{mg}$ of drugloaded powder MOFs containing $5 \mathrm{mg}, 4,9 \mathrm{mg}$ and 5,5 $\mathrm{mg}$ of diclofenac sodium in $\gamma$-KCD, $\gamma$-NaCD and $\gamma$-FeCD, respectively were placed into a cellulose membrane dialysis bag $(25 \times 16 \mathrm{~mm})$, which was previously hydrated containing $1 \mathrm{~mL}$ of release media, and placed in a vessel containing $50 \mathrm{~mL}$ of release media, according to sink conditions, at $37^{\circ} \mathrm{C}$ under constant stirring of $100 \mathrm{rpm}$, in triplicate. In vitro release was performed in three different media with different $\mathrm{pH}$ to simulate one of the parameters to simulate the physiological conditions. Medium 1 (pH 1.2) was prepared with $0.2 \mathrm{~N} \mathrm{HCl}$ containing $1.5 \%(\mathrm{w} / \mathrm{v})$ polysorbate 80 up to $2 \mathrm{~h}$ to simulate the gastric fluid; thereafter, the drugloaded powder MOFs contained in a cellulose membrane dialysis bag were transferred to medium 2, PBS (pH 7.4) to simulate intestinal fluid. Then, medium 2 was $\mathrm{pH}$-adjusted to medium 3 over $4 \mathrm{~h}$, which PBS (pH 6.8), without the need to transfer the MOF to another medium for up to $24 \mathrm{~h}$ to simulate colonic fluid [21], to reproduce almost all of the physiological conditions encountered during the journey of a delivery system following oral administration.

The determination of the amount of DFNa released in the medium was determined by UV spectroscopy, at $275 \mathrm{~nm}$. UV-Vis absorption data were recorded within the wavelength range of $200-500 \mathrm{~nm}$ using an Agilent Technologies Cary 60 dual beam spectrophotometer fitted with a fibre-optic coupler equipped with an immersion probe. This allows a $4 \mathrm{~mm}$ thick solution to be analysed, making the technique sensitive to the DFNa molecules delivered in aqueous solutions, without the need to remove or replace dissolution medium at each point. The acquisition scan rate was $300 \mathrm{~nm} \cdot \mathrm{min}^{-1}$ and a full spectrum was recorded in $60 \mathrm{~s}$.

The mechanism of drug release was analysed by fitting the release data with empirical equations following different mathematical models (Zero Order, First Order, Korsmeyer-Peppas, Higuchi, Weibull and Hixson-Crowell) with Sigma Plot 10.0 software.

\subsection{In vivo experiments}

The experimental protocol was performed in accordance with the Guide for the Care and Use of Laboratory Animals and the Ethical
Principles for Animal Experimentation established by the Brazilian Committee for Animal Experimentation (COBEA). This investigation was approved by the animal experimentation ethics committee of the School of Pharmaceutical Sciences/UNESP (Protocol CEUA/FCF/Car N ${ }^{\circ}$ 41/2016), and complied with international laws. The evaluation of anti-inflammatory activity in vivo was performed on male Swiss mice $(30-35 \mathrm{~g})$. Mice were randomised into 5 groups $(\mathrm{n}=5)$ and maintained at $25^{\circ} \mathrm{C}$ and dark/light cycle $(12 / 12 \mathrm{~h})$.

Anti-inflammatory activity was measured using carrageenan-induced mouse paw oedema assay. Oedema was induced by intraplantar injection of $100 \mu \mathrm{L}$ of $1 \%$ solution of carrageenan into the paw of each mice of all groups. Groups received the following treatments about $1 \mathrm{~h}$ after inflammation was induced: (1) diclofenac sodium $(100 \mathrm{mg} / \mathrm{kg}$ body weight), (2) without treatment; (3) $\gamma$-FeCD (182 mg/kg), (4) $\gamma$ $\mathrm{KCD}(200 \mathrm{mg} / \mathrm{kg})$; or (5) $\gamma-\mathrm{NaCD}(204 \mathrm{mg} / \mathrm{kg})$. It is important to note that the dose of the drug was the same between the drug alone and the MOF systems $(100 \mathrm{mg} / \mathrm{kg})$; for this, the entrapment efficiency (\%) of each MOF was considered. Therefore, the dose of drug was maintained to not interfere with in vivo experiments and consequently enable to compare the anti-inflammatory effect in different groups. All samples were dispersed in purified water and administered orally by gavage. Paw thickness was measured with a digital calliper just before the carrageen injection, that is, at " $0 \mathrm{~h}$ ", and then at 4,6 and $24 \mathrm{~h}$ after carrageen injection. Data were plotted using GraphPad Prism v. 6.0 and the ANOVA test was performed followed by Dunnett's test ( $\alpha=0.05$ ). Increase in paw thickness was measured as the difference in paw thickness at " $0 \mathrm{~h}$ " and paw thickness at respective hours. The inhibition of oedema was calculated by the following Eq. (3):

$\%$ Inhibition $=1-\left[\frac{(\text { Test }- \text { Basal })}{(\text { Control-Basal })}\right] x 100$

\section{Results and discussion}

Particles of three types of $\gamma$-CD-MOFs: $\gamma$-KCD, $\gamma$-NaCD and $\gamma$-FeCD, either with a crystalline porous framework, were obtained using the vapour diffusion method with methanol. The $\gamma$-KCD and $\gamma$-NaCD synthesis had been previously reported [10], so we now developed a new material using the same synthetic route based on iron metal as a node and $\gamma$-cyclodextrin as an organic linker, named $\gamma$-FeCD.

This method of synthesis occurs because the methanol vapour, which is more volatile, upon contact with the aqueous solution of reagents, through diffusion, gradually decreases the solution polarity and consequently decreases the solubility of the sample, leading to slow crystal formation [13].

The link between the metal and $\gamma$-cyclodextrin occurs by coordinate bonds, as the-O-position in the $\gamma$-cyclodextrin allows the formation of an ether crown, which facilitates the electron donation to the metals and the formation of bonds $[10,13]$.

The vapour diffusion method has some advantages compared to other usual methods for the synthesis of MOFs, such as the simplicity of the synthesis that does not need heating, either solvothermal or microwave. In addition, the yield of this type of synthesis is very high.

The yield obtained for the synthesised $\gamma$-CD-MOFs was very similar to the values reported in the literature by Smaldone and collaborators [10], around $70 \%$, which shows the efficiency of the synthesis performed. Yields of $\gamma$-KCD and $\gamma$-NaCD were $70 \%$ and $72 \%$, respectively. For $\gamma$-FeCD, we obtained the yield of $62 \%$, showing the efficiency of the synthesis process, and did not use a class of alkaline/alkaline earth metals, but rather a transition metal.

Fig. 2 shows the XRD diffractograms of all $\gamma$-CD-MOFs and free $\gamma$ $\mathrm{CD}$. The samples showed similar peaks with pure cyclodextrin, proving the presence of the organic linker, cyclodextrin, in the samples formed. The XRD patterns confirmed the crystalline morphologies of all of the samples synthesised with different metals, including the evident peaks 


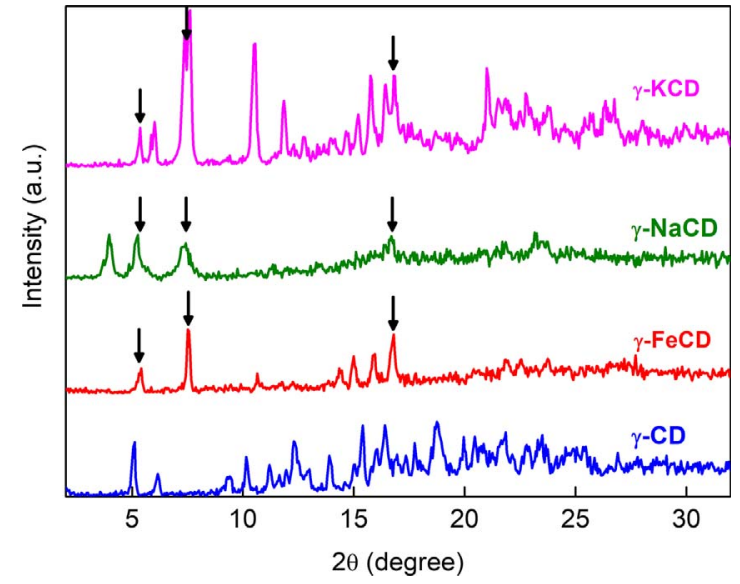

Fig. 2. XRD crystallinity patterns of $\gamma$-KCD, $\gamma$-NaCD, $\gamma$-FeCD and free $\gamma$-CD.

of $\gamma$-CD-MOFs as $5.3^{\circ}, 7.4^{\circ}$ and $16.7^{\circ}$, indicated with arrows in Fig. 2, which are in good agreement with previously reported data $[10,13,14,22]$.

Fig. 3 shows the IR spectra of the three different $\gamma$-CD-MOFs and free $\gamma-C D$, in the spectral range between 400 and $4000 \mathrm{~cm}^{-1}$ and is in agreement with that previously reported in the literature [18]. An important stretching band derived from the $\mathrm{CH}_{2}$ group was observed (2850-3000 $\mathrm{cm}^{-1}$ ), which became narrower in $\gamma$-CD-MOF, accounting for the angular chain deformation, which can be related to the molecular conformational changed after the formation of the metal-cyclodextrin bond. At $1020-1150 \mathrm{~cm}^{-1}$, a stretching vibration mode derived from the -C-O-C group can be observed. However, for free $\gamma$-CD samples, the peak is narrower when compared to the $\gamma$-CD-MOFs due to cross-linking between the units of 1,4-linked-alpha-D-glucopyranosyl residues from cyclodextrins in the $\gamma$-CD-MOF.

In order to evaluate the thermal stability of $\gamma$-CD-MOFs, we used thermogravimetric analysis (TGA), as shown in Fig. 4. We also examined their thermal stability of guest-free samples before and after activation. TGA traces show the retention of solvents by $\gamma$-KCD, and the solvent evacuation, with thermal stability of the sample under $200{ }^{\circ} \mathrm{C}-210{ }^{\circ} \mathrm{C}$. The stability to heating of $\gamma$-KCD activated is shows in its TGA curve, with the retention of mass up to $210^{\circ} \mathrm{C}$, losing $13 \%$ in mass at approximately $100{ }^{\circ} \mathrm{C}$, probably corresponding to the loss of water. An analogous experiment with $\gamma$-NaCD showed a similar behaviour, with thermal stability of the sample under $205^{\circ} \mathrm{C}-215^{\circ} \mathrm{C}$ after activation and the loss of water confirmed with a small drop (8\%) in mass at approximately $100^{\circ} \mathrm{C}$. Finally, TGA curves of activated $\gamma$-FeCD shows that thermal degradation occurs at temperatures over $135^{\circ} \mathrm{C}$.

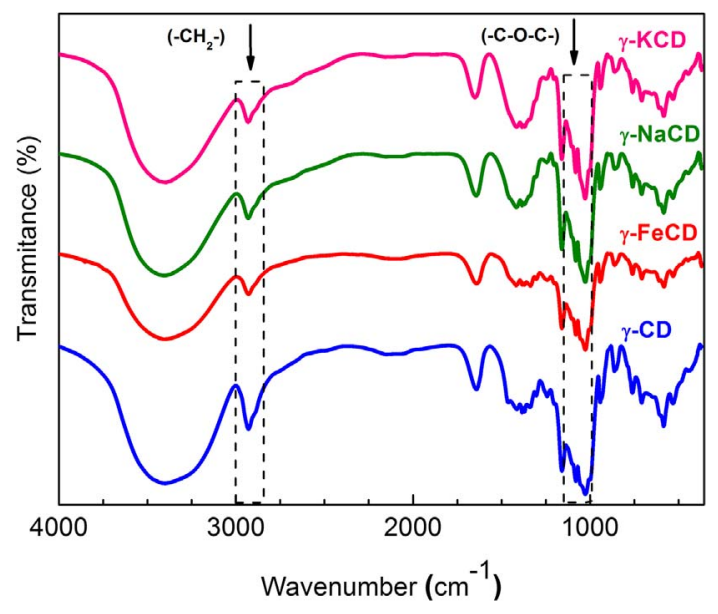

Fig. 3. IR patterns of $\gamma$-KCD, $\gamma-\mathrm{NaCD}, \gamma-\mathrm{FeCD}$ and free $\gamma$-CD.
Therefore, the results of TGA indicate that the loss in mass before activation is attributed to the expulsion of interstitial solvent trapped in the framework and confirmed that the pores are unlocked for drug incorporation.

The pore sizes of activated $\gamma$-CD-MOFs were calculated from nitrogen adsorption-desorption. The $\gamma$-KCD sample exhibits a pore size of $24.13 \AA$, while for $\gamma$-NaCD this is $32.86 \AA$ and for $\gamma$-FeCD this is $30.19 \AA$, indicating the mesoporosities of these materials, contributing to their utility as drug delivery devices. The estimated bulk density (void space) is $0.81 \mathrm{~g} . \mathrm{cm}^{-3}(47 \%), 0.86 \mathrm{~g} . \mathrm{cm}^{-3}(46.3 \%)$ and $0.67 \mathrm{~g} . \mathrm{cm}^{-3}(58.94 \%)$ for $\gamma$-KCD, $\gamma$-NaCD and $\gamma$-FeCD, respectively, which is almost the same as that previously reported in the literature [10].

SEM images acquired from the dried samples revealed interesting microstructural attributes elucidating the structural organisation of all particles. Fig. 5a shows $\gamma$-KCD crystals before the activation with solvent surrounding the particles. After the activation, with the $\mathrm{CHCl}_{2-}$ exchange method, the remaining free linker and solvent into the pores are removed and the crystals and their surfaces are free (Fig. 5b). Fig. 5c shows a cubic unit crystalline of $\gamma$-KCD. The $\gamma$-NaCD before and after the activation is shown in Fig. $5 \mathrm{~d}$ and e, respectively. It is possible to observe the presence of surface pores in the active material (Fig. 5e and f). Fig. $5 \mathrm{~g}$ shows $\gamma$-FeCD crystals before activation, while Fig. $5 \mathrm{~h}$ and $i$ after activation, which shows the structure of a pack of laminate packed together, that were built from very small cubic structures. The geometry of $\gamma$-FeCD could be justified by extension of the structural arrangement of pores only in one direction, which can provide a drug release behaviour that discriminates from other materials. The morphological orders of the samples with the visible pores presented enhance the application of the material as a drug carrier.

The cytotoxicity assay was analysed to obtain a preliminary safety profile of $\gamma$-CD-MOFs applied in human cells. It is noteworthy that the $\gamma$ CD-MOFs had neutral or slightly basic pH in PBS and DMEM, which was extremely important to know whether the material would be able to perform the cytotoxicity test because the $\mathrm{pH}$ values are within the range of $\mathrm{pH}$ of cellular survival, not interfering in the results of cellular viability and avoiding a false result (Table 1, Supporting Information). Two cell lines were chosen: Caco-2 human colon adenocarcinoma and HepG2 human hepatoma cell lines. The dose-response relationship for the cytotoxic effect of $\gamma$-CD-MOF to Caco-2 and HepG2 cells was assessed after $24 \mathrm{~h}$ of treatment. The toxicity study demonstrated that there was no significant difference $(p>0.05)$ in cell viability in cells exposed to the different $\gamma$-CD-MOFs in the concentrations assessed. Thus, $\gamma$-CD-MOFs were found to be not cytotoxic (cell viability mean $>100 \%$ ) up to a concentration of $2000 \mu \mathrm{g} / \mathrm{mL}$ to both mammalian cell lines tested, as shown in Fig. 6. Furthermore, the percentage of cell viability was not altered in the various concentrations assessed. This is another indication that the treatments with $\gamma$-CD-MOFs did not affect the cells behaviour. Also, the result was similar for all of the different $\gamma$-CD-MOFs assessed: $\gamma$-KCD, $\gamma$-NaCD and $\gamma$-FeCD. This behaviour is similar to that obtained by Tofzikovskaya and collaborators [23], which assessed the cytotoxicity of $\gamma$-CDs in various cells lines and observed the absence of a toxic effect. Considering the absence of cytotoxicity, the authors expect that it is safe for medical use.

It is important to emphasise that the higher concentration assessed $(2000 \mu \mathrm{g} / \mathrm{mL})$ corresponds to the limit of $\gamma$-CD-MOF dispersion in DMEM. In higher concentrations, precipitation was visualised.

The entrapment efficiency (\%) of DFNa in the $\gamma$-KCD, $\gamma$-NaCD and $\gamma$ FeCD particles were $50 \pm 0.015,49 \pm 0.109$, and $55 \pm 0.140$, respectively. Previous data reported in the literature of $\gamma$-CD-MOF exhibited low values of drug adsorption with other anti-inflammatories, e.g. $0.30 \% \pm 0.03$ of Paracetamol, $9.83 \% \pm 1.07$ of Salicylic acid and $12.10 \% \pm 0.11$ of Flurbiprofen [18]. It is noteworthy that the entrapment efficiency obtained in this study proved that the value obtained is independent of the metal used to compose the metal-organic framework. These results confirmed the good capacity of the $\gamma$-CD-MOF to incorporate a high amount of drug; this finding can be extend to other 
a)

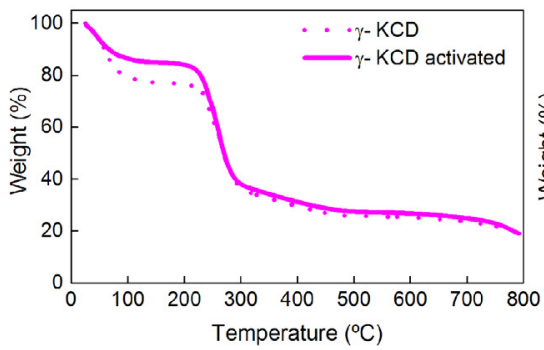

b)

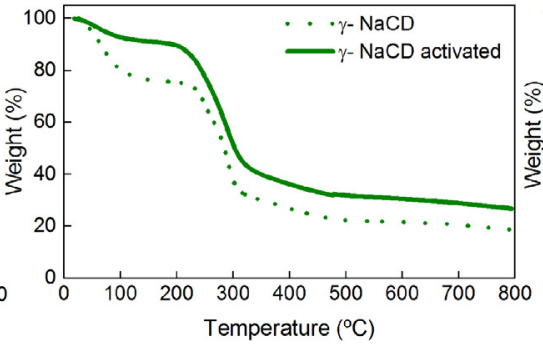

c)

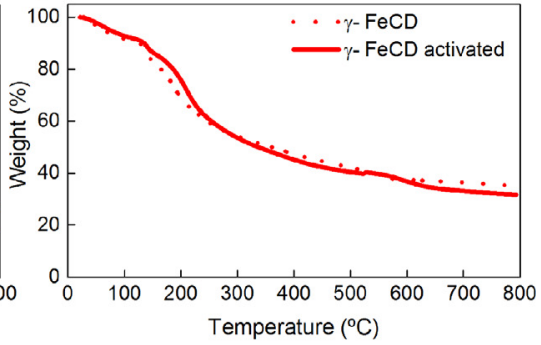

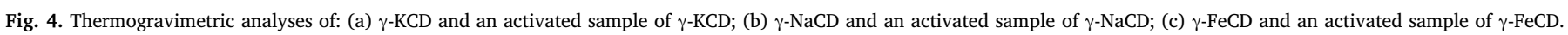
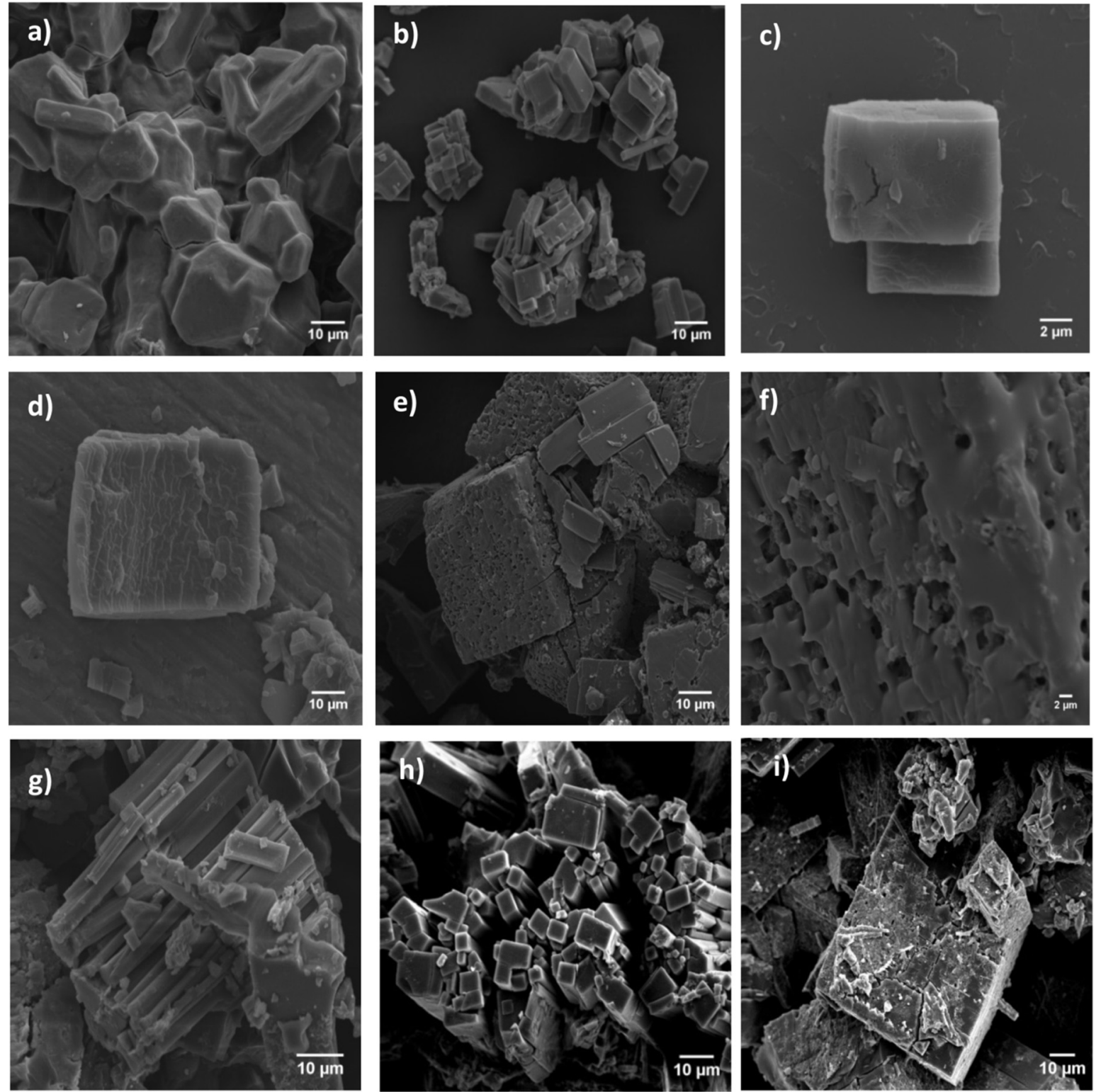

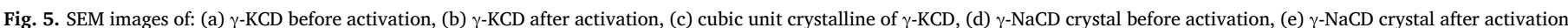
and (f) its zoom that shown the surface pores, (g) laminate packed of $\gamma$-FeCD before activation, (h) $\gamma$-FeCD after activation, (i) $\gamma$-FeCD after activation with the surface pores.

drugs, but may cause different release mechanisms and an improved effect on the drug delivery system.

The in vitro release profile of DFNa from different $\gamma$-CD-MOF and free drugs are presented in Fig. 7. In the first two hours of the release test, the samples $\gamma$-KCD $\gamma$-NaCD and $\gamma$-FeCD released only $23 \%, 22 \%$ and $20 \%$ of DFNa, respectively, demonstrating reduced release rates in acid media ( $\mathrm{pH}$ 1.2). In contrast, free drugs displayed a burst release in which above $70 \%$ of the drug was released in the first $0.5 \mathrm{~h}$ of the test, and achieved a complete release after $5 \mathrm{~h}$.

In PBS (pH 7.4), the samples $\gamma$-KCD $\gamma$-NaCD and $\gamma$-FeCD released $33 \%, 39 \%$ and $42 \%$, respectively, in a six hour assay. The constant drug release was maintained due to the formation of a more compact 

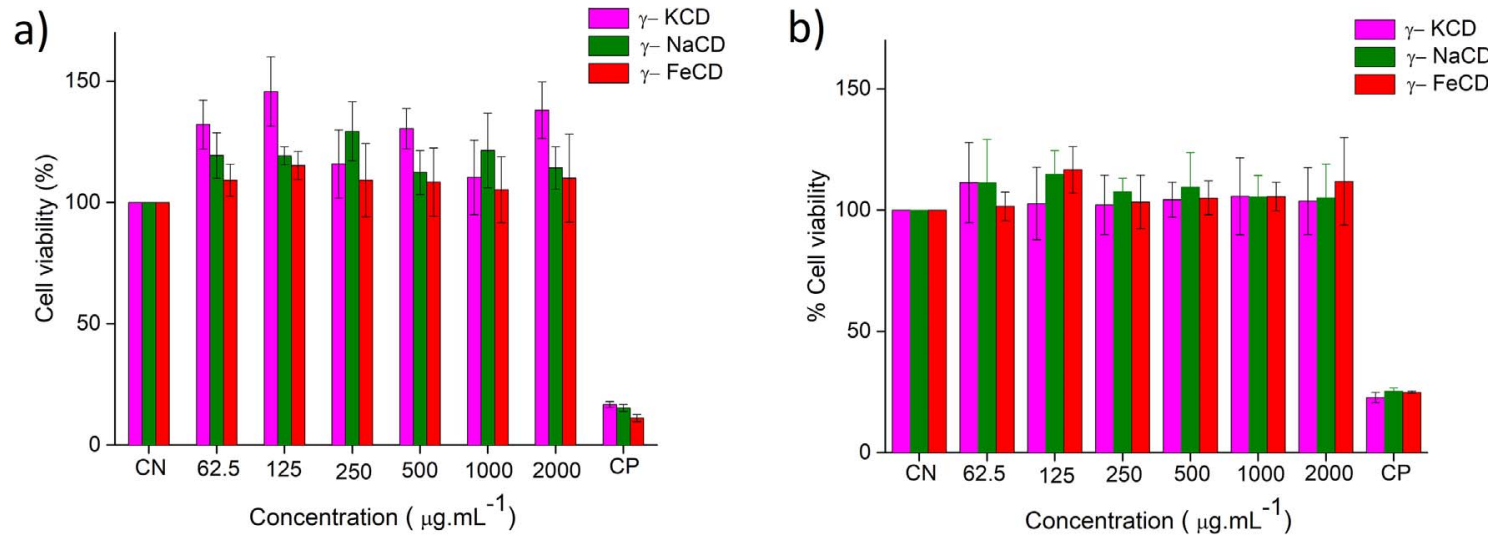

Fig. 6. Cytotoxic effect of $\gamma$-CD-MOFs at concentration $62.5 \mu \mathrm{g} / \mathrm{mL}-2000 \mu \mathrm{g} / \mathrm{mL}$ on: (a) HepG2 cell lines and (b) Caco-2 cell lines.

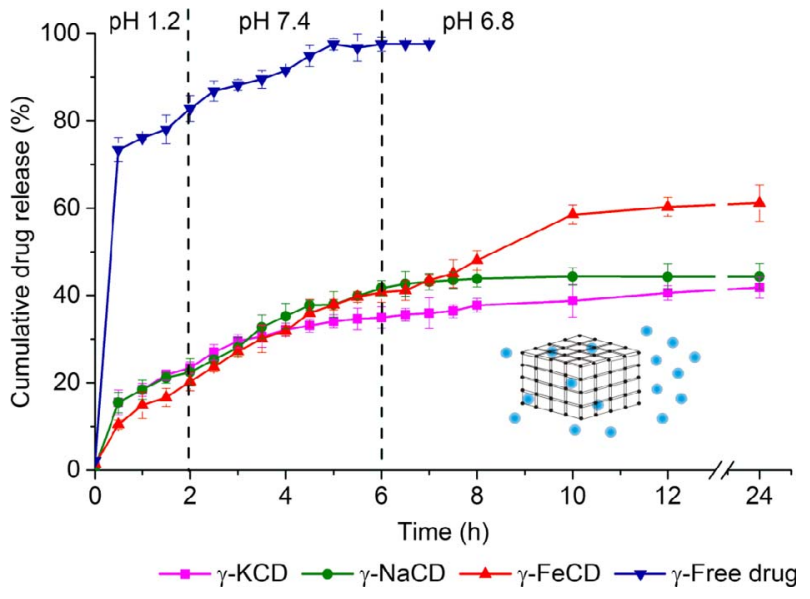

Fig. 7. Cumulative release rates of diclofenac sodium in different simulated biological fluids: $\mathrm{HCl} 0.2 \mathrm{~N}(\mathrm{pH} 1.2)$ to simulate the gastric fluid, phosphate buffer solution (pH 7.4) to simulate the intestinal fluid and phosphate buffer solution ( $\mathrm{pH} 6.8$ ) to simulate the colonic fluid.

structure with high density, which should restrict the inflow of liquid and, consequently, reduce the drug diffusion capacity for the dissolution medium, promoting the greater control of release. This behaviour is in agreement with the SEM images in which all samples presented a rigid and compact structure, which occurs due to the use of the same organic linker, $\gamma$-cyclodextrin. Already in PBS ( $\mathrm{pH}$ 6.8), the first important observation is that regardless of the $\gamma$-CD-MOFs used, the amount of drug release was similar until $8 \mathrm{~h}$ of the test $(\mathrm{p}<0.05)$. After this time, the $\gamma$-FeCD sample shows a remarkable increase in the amount of DFNa released ( $p>0.05$ ), and the cumulative percentage of drug release within $24 \mathrm{~h}$ reached approximately $63 \%$ in the case of $\gamma$ FeCD, while it was around 41 and $42 \%$ for $\gamma$-KCD and $\gamma$-NaCD, respectively. This particular behaviour of $\gamma$-FeCD can be attributed to the higher pore volume this sample, which provides more space for the solvent to entrain and release the drug. Moreover, the laminated morphology of the $\gamma$-FeCD was also favoured for less control over drug release, having a different release behaviour of the samples composed of other metals that present a cubic structure, as can be seen in the SEM images.

These results indicate the capacity of $\gamma$-CD-MOFs to control the DFNa release throughout the gastrointestinal tract, which demonstrates the applicability of the metal-organic framework on the design of controlled release systems or in colon-specific drug delivery systems.

The data of in vitro DFNa release correlated better with the Weibull mathematical model for all samples (higher $\mathrm{R}^{2}$ ) (Table 1 ). The release exponent value $b(>1)$ suggested that diclofenac sodium release from different $\gamma$-CD-MOF followed a complex mechanism of release, in which
Table 1

Kinetic models used for analysis of DFNa release from different $\gamma$-CD-MOFs and overall correlation coefficient $\left(\mathrm{R}^{2}\right)$ and release constant $(\mathrm{K})$.

\begin{tabular}{lllll}
\hline Release model & & $\gamma$-KCD & $\gamma$-NaCD & $\gamma$-FeCD \\
\hline Zero order & $\mathrm{K}$ & 0.2686 & 0.1907 & 0.1979 \\
\multirow{3}{*}{ Higuchi } & $\mathrm{R}^{2}$ & 0.7076 & 0.6803 & 0.8418 \\
Korsmeyer-Peppas & $\mathrm{K}$ & 10.6080 & 11.4861 & 14.1490 \\
& $\mathrm{R}^{2}$ & 0 & 0 & 0.8137 \\
First order & $\mathrm{K}$ & 23.1911 & 24.2127 & 21.8674 \\
& $\mathrm{R}$ & 0.9642 & 0.9098 & 0.9204 \\
Hixson-Crowell & $\mathrm{n}$ & 0.1832 & 0.2003 & 0.3290 \\
\multirow{3}{*}{ Weibull } & $\mathrm{K}$ & 0.0494 & 0.0619 & 0.0862 \\
& $\mathrm{R}$ & 0 & 0 & 0.8281 \\
& $\mathrm{~K}$ & 0.0146 & 0.0196 & 0.0250 \\
& $\mathrm{R}$ & 0 & 0 & 0.7598 \\
& $\mathrm{~K}$ & 0.1594 & 0.1127 & 0.1678 \\
& $\mathrm{R}^{2}$ & 0.9937 & 0.9887 & 0.9941 \\
& $\mathrm{~b}$ & 2.4658 & 2.7263 & 7.2052 \\
\hline
\end{tabular}

various processes, such as diffusion, swelling and erosion can occur simultaneously during drug release. These findings supported our concept of the formation of a solid metal-organic framework, which had the capacity to perform the drug release by different mechanisms through pores.

The diffusion capacity is a constant of proportionality, which can be established as a function of concentration or physical variables that are related to the structure of the material, such as the existence of free volume between the polymer chains, which allows the drug diffusion [24]. Therefore, the release of drugs from $\gamma$-CD-MOFs is directly related to drug desorption from the formed pores, drug diffusion through the frameworks, erosion of the material or even the combination of these processes. It is possible to emphasise their potential as drug delivery carriers and that they offer non-invasive routes of administration such as oral routes.

The study of in vivo anti-inflammatory activity demonstrated that all samples had the same inhibition of inflammation compared to the control at 4 and $6 \mathrm{~h}$, except for the sample $\gamma$-FeCD at $6 \mathrm{~h}(\mathrm{p}<0.01)$ (Fig. 8). Moreover, the effect of $\gamma$-FeCD was active for up to $24 \mathrm{~h}$ and was higher than the other materials tested ( $\gamma$-KCD and $\gamma$-NaCD). This phenomenon can be explained by the ability of the material to control the release of DFNa, unlike free DFNa, which is readily free for dissolution in fluids. Besides that, it is important to note that in vitro release and in vivo experiments could have a few differences explained by the animal having a metabolic complex that should be influenced by the presence of surface pores and three-dimensional structure of systems that could facilitate the drug diffusion during the first hours and contribute to the biological effect, which was the same anti-inflammatory effect between $\gamma$-KCD, $\gamma$-NaCD and drug alone.

The results obtained in the efficacy studies corroborate with the in 


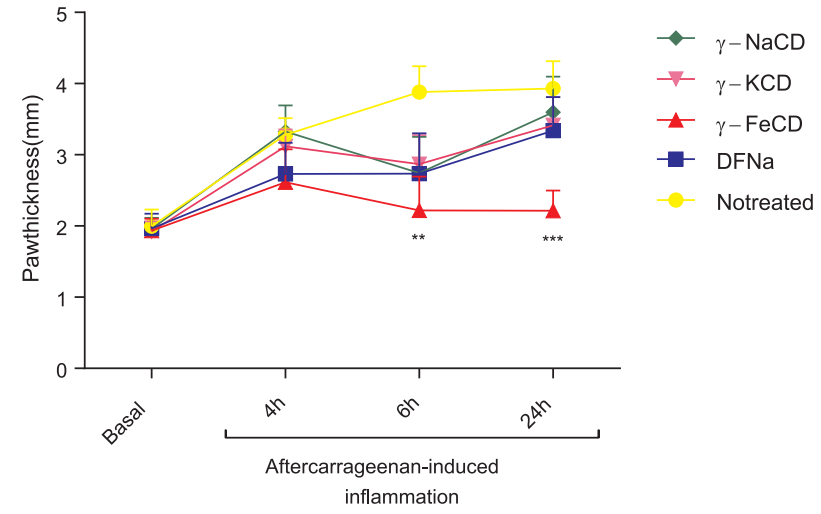

Fig. 8. Anti-inflammatory activity of the DFNa loaded MOFs ( $\gamma$-FeCD, $\gamma$-NaCD and $\gamma$ KCD), DFNa as positive control and untreated in mices. Notes: The data are represented mean and standard deviation of results on five mice. The statistical significance of paw thickness, was analysed using variance analysis by Dunnett's multiple comparison test; p $<<0.01 ;{ }^{* * *} \mathrm{p}<0.001$.

vitro release, in which the $\gamma$-FeCD sample has a release rate that is higher than $\gamma$-KCD and $\gamma$-NaCD. Thus, this difference presented by $\gamma$ FeCD can contributed to a greater effect on the anti-inflammatory activity, due to a faster release of the drug.

It is important to highlight that in $24 \mathrm{~h}$, the performances of the inhibition of oedema were $30.4 \%, 88.7 \%, 26.8 \%$ and $17.3 \%$ for DFNa, $\gamma$-FeCD, $\gamma$-KCD and $\gamma$-NaCD, respectively. At this time, $\gamma$-FeCD indicated a statistically significant decrease in paw thickness, having improved effects on anti-inflammatory response; the mean difference was found for DFNa ( $\mathrm{p}<0.001) \cdot \gamma$-CD-MOF-loaded DFNa significantly decreased the inflammatory oedema induced by carrageenan.

All of these characteristics make $\gamma$-CD-MOFs promising materials for controlled drug release systems applied to oral delivery. In vivo toxicity, efficiency, and pharmacokinetics of the drug-loaded $\gamma$-CD-MOFs are important steps that demand full investigation to estimate the actual performances of $\gamma$-CD-MOFs [25].

\section{Conclusion}

$\gamma$-CD-MOFs were successfully synthesised by an optimised vapour diffusion method, and, thus, crystals with uniform structure and size suitable for oral delivery were obtained. The anti-inflammatory drug was successfully entrapped within porous $\gamma$-CD-MOFs, achieving exceptional payloads and controlled release, simulating the gastrointestinal tract, suggesting the applicability of these materials on the design of controlled release systems or in colon-specific drug delivery systems with a greater emphasis on $\gamma$-FeCD. This has a greater effect on the in vivo anti-inflammatory activity, due to the faster release of the drug. Our conclusion is that a promising oral drug delivery system was obtained, with more marked characteristics than those studied until now.

\section{Author contributions}

Marina P. Abuçafy performed the synthesis, physicochemical characterisation, in vitro assay, drug encapsulation test, in vitro drug release and in vivo anti-inflammatory assay. Bruna G. Chiari-Andréo performed the in vitro toxicity assay. Bruno F-Santos, Aline M. do Santos and Marlus Chorilli performed the in vivo anti-inflammatory assay. Bruno L. Caetano performed the physicochemical characterisation, in vitro drug release, and in vivo anti-inflammatory assay, Leila A. Chiavacci supervised Marina P. Abuçafy and developed the systems to coordinate the research. All authors assisted in drafting the paper and approved the final version.

\section{Acknowledgments}

The authors thank São Paulo Research Foundation - FAPESP (2014/ 10888-1), National Council for Scientific and Technological Development - CNPq, and Coordination for the Improvement of Higher Education Personnel - CAPES for the financial support. They would also like to thank Professors Celso V. Santilli, Sandra H. Pulcinelli, Regina Maria Barretto Cicarelli and Vera Lucia Borges Isaac (UNESP/ Araraquara) for allowing them to use their laboratory during the characterisation of the materials and for the donation of cells.

\section{Appendix A. Supplementary material}

Supplementary data associated with this article can be found, in the online version, at https://doi.org/10.1016/j.ejpb.2018.02.009.

\section{References}

[1] H. Deng, C.J. Doonan, H. Furukawa, R.B. Ferreira, J. Towne, C.B. Knobler, B. Wang, O.M. Yaghi, Multiple functional groups of varying ratios in metal-organic frameworks, Science (80-.) 327 (2010) 846-850.

[2] P. Horcajada, C. Serre, G. Maurin, N.A. Ramsahye, F. Balas, M. Vallet-Regi, M. Sebban, F. Taulelle, G. Férey, Flexible porous metal-organic frameworks for a controlled drug delivery, J. Am. Chem. Soc. 130 (2008) 6774-6780.

[3] A. Dhakshinamoorthy, H. Garcia, Metal-organic frameworks as solid catalysts for the synthesis of nitrogen-containing heterocycles, Chem. Soc. Rev. 43 (2014) 5750-5765.

[4] Q. Yang, C. Zhong, Molecular simulation of carbon dioxide/methane/hydrogen mixture adsorption in metal-organic frameworks, J. Phys. Chem. B 110 (2006) 17776-17783.

[5] T.B. Lee, D. Kim, D.H. Jung, S.B. Choi, J.H. Yoon, J. Kim, K. Choi, S.-H. Choi, Understanding the mechanism of hydrogen adsorption into metal organic frameworks, Catal. Today 120 (2007) 330-335, http://dx.doi.org/10.1016/j.cattod. 2006.09.030.

[6] T. Rodenas, I. Luz, G. Prieto, B. Seoane, H. Miro, A. Corma, F. Kapteijn, F.X.L. i Xamena, J. Gascon, Metal-organic framework nanosheets in polymer composite materials for gas separation, Nat. Mater. 14 (2015) 48-55.

[7] H. Chen, Y. Zheng, J.-H. Jiang, H.-L. Wu, G.-L. Shen, R.-Q. Yu, An ultrasensitive chemiluminescence biosensor for cholera toxin based on ganglioside-functionalized supported lipid membrane and liposome, Biosens. Bioelectron. 24 (2008) 684-689, http://dx.doi.org/10.1016/j.bios.2008.06.031.

[8] P. Horcajada, R. Gref, T. Baati, P.K. Allan, G. Maurin, P. Couvreur, G. Ferey, R.E. Morris, C. Serre, Metal-organic frameworks in biomedicine, Chem. Rev. 112 (2011) 1232-1268.

[9] P. Horcajada, C. Serre, M. Vallet-Regí, M. Sebban, F. Taulelle, G. Férey, Metal-organic frameworks as efficient materials for drug delivery, Angew. Chem. 118 (2006) 6120-6124.

[10] R.A. Smaldone, R.S. Forgan, H. Furukawa, J.J. Gassensmith, A.M.Z. Slawin, O.M. Yaghi, J.F. Stoddart, Metal-organic frameworks from edible natural products, Angew. Chem. Int. Ed. 49 (2010) 8630-8634.

[11] J. Chen, K. Li, L. Chen, R. Liu, X. Huang, D. Ye, Conversion of fructose into 5 hydroxymethylfurfural catalyzed by recyclable sulfonic acid-functionalized metal-organic frameworks, Green Chem. 16 (2014) 2490-2499, http://dx.doi.org/10. 1039/C3GC42414F.

[12] Y. Yang, R. Lin, L. Ge, L. Hou, P. Bernhardt, T.E. Rufford, S. Wang, V. Rudolph, Y. Wang, Z. Zhu, Synthesis and characterization of three amino-functionalized metal-organic frameworks based on the 2-aminoterephthalic ligand, Dalt. Trans. 44 (2015) 8190-8197, http://dx.doi.org/10.1039/C4DT03927K.

[13] R.S. Forgan, R.A. Smaldone, J.J. Gassensmith, H. Furukawa, D.B. Cordes, Q. Li, C.E. Wilmer, Y.Y. Botros, R.Q. Snurr, A.M.Z. Slawin, Nanoporous carbohydrate metal-organic frameworks, J. Am. Chem. Soc. 134 (2011) 406-417.

[14] Y. Furukawa, T. Ishiwata, K. Sugikawa, K. Kokado, K. Sada, Nano- and microsized cubic gel particles from cyclodextrin metal-organic frameworks, Angew. Chem. Int. Ed. 51 (2012) 10566-10569, http://dx.doi.org/10.1002/anie.201204919.

[15] J.J. Gassensmith, H. Furukawa, R.A. Smaldone, R.S. Forgan, Y.Y. Botros, O.M. Yaghi, J.F. Stoddart, Strong and reversible binding of carbon dioxide in a green metal-organic framework, J. Am. Chem. Soc. 133 (2011) 15312-15315.

[16] H. Dodziuk, Cyclodextrins and Their Complexes: Chemistry, Analytical Methods, Applications, John Wiley \& Sons, 2006.

[17] P. Sher, G. Ingavle, S. Ponrathnam, P. Poddar, A.P. Pawar, Modulation and optimization of drug release from uncoated low density porous carrier based delivery system, AAPS PharmSciTech. 10 (2009) 547-558.

[18] B. Liu, H. Li, X. Xu, X. Li, N. Lv, V. Singh, J.F. Stoddart, P. York, X. Xu, R. Gref, J. Zhang, Optimized synthesis and crystalline stability of $\gamma$-cyclodextrin metal-organic frameworks for drug adsorption, Int. J. Pharm. 514 (2016) 212-219, http:// dx.doi.org/10.1016/j.ijpharm.2016.09.029.

[19] H. Li, M. Eddaoudi, M. O'Keeffe, O.M. Yaghi, Design and synthesis of an exceptionally stable and highly porous metal-organic framework, Nature 402 (1999) 276-279 http://www.nature.com/nature/journal/v402/n6759/suppinfo/ 402276a0_S1.html. 
[20] N.S. Santos-Magalhaes, A. Pontes, V.M.W. Pereira, M.N.P. Caetano, Colloidal carriers for benzathine penicillin G: nanoemulsions and nanocapsules, Int. J. Pharm. 208 (2000) 71-80.

[21] P. Bertocchi, E. Antoniella, L. Valvo, S. Alimonti, A. Memoli, Diclofenac sodium multisource prolonged release tablets - A comparative study on the dissolution profiles, J. Pharm. Biomed. Anal. 37 (2005) 679-685, http://dx.doi.org/10.1016/j. jpba.2004.11.041.

[22] H. Li, N. Lv, X. Li, B. Liu, J. Feng, X. Ren, T. Guo, D. Chen, J. Fraser Stoddart, R. Gref, J. Zhang, Composite CD-MOF nanocrystals-containing microspheres for sustained drug delivery, Nanoscale 9 (2017) 7454-7463, http://dx.doi.org/10.
1039/C6NR07593B.

[23] Z. Tofzikovskaya, A. Casey, O. Howe, C. O'Connor, M. McNamara, In vitro evaluation of the cytotoxicity of a folate-modified $\beta$-cyclodextrin as a new anti-cancer drug delivery system, J. Incl. Phenom. Macrocycl. Chem. 81 (2015) 85-94, http:// dx.doi.org/10.1007/s10847-014-0436-0.

[24] L.T. Fan, S.K. Singh, Controlled release: A Quantitative Treatment, Springer, Berlin, New York, 1989.

[25] R.C. Huxford, J. Della Rocca, W. Lin, Metal-organic frameworks as potential drug carriers, Curr. Opin. Chem. Biol. 14 (2010) 262-268. 